\title{
COMPARAÇÃO ENTRE O ÍNDICE DE QUALIDADE DA ÁGUA (IQA) COM O PROTOCOLO DE AVALIAÇÃO RÁPIDA DE HABITATS NO CÓRREGO LISO, MUNICÍPIO DE SÃO SEBASTIÃO DO PARAÍSO, MINAS GERAIS
}

\author{
Alexandre Cardoso Souza ${ }^{1}$ \\ Thainá Desiree Franco dos Reis ${ }^{2}$ \\ Odila Rigolin de Sá ${ }^{3}$
}

\begin{abstract}
RESUMO
Nas últimas décadas, estudos sobre conservação e monitoramento de habitats mostraram-se em constante e importante crescimento. Novas técnicas de monitoramento foram desenvolvidas e são consideradas importantes no meio científico, justamente pela facilidade, rapidez e precisão dos resultados apresentados. Este trabalho teve por objetivo utilizar o Protocolo de Avaliação Rápida da Diversidade de Habitats, proposto por Callisto et al. (2002), como ferramenta para uma avaliação visual, rápida e qualitativa, visando avaliar a estrutura e funcionamento dos ecossistemas e comparar com o Índice de Qualidade da Água (IQA), no sentido de contribuir para o manejo e conservação dos mesmos. As coletas de dados foram realizadas nos dias 20 e 23 de março de 2014, em seis pontos de amostragem no Córrego Liso, localizado no município de São Sebastião do Paraíso, Minas Gerais. Percebe-se que no Córrego Liso há uma forte ação antrópica, e com a aplicação do protocolo foi possível diagnostica-lo como "impactado" segundo a pontuação final de todos os pontos estudados que variaram de 26 a 31. De acordo com o relatório publicado pelo Instituto Mineiro de Gestão das Águas (IGAM) o referido Córrego apresentou um IQA de 25,1 no $4^{\circ}$ trimestre de 2013 , e no $1^{\circ}$ trimestre de 2014 apresentou IQA de 34,6, no entanto permanecendo como "ruim", o que se confirma na metodologia do protocolo rápido, mostrando sua eficiência para diagnosticar ambientes lóticos. Necessita-se ainda aplicar medidas mitigadoras urgentes para que o córrego não piore ainda mais sua qualidade limnológica, agravando cada vez mais o sua classificação perante o IQA.
\end{abstract}

PALAVRAS-CHAVE: Habitats, Ambientes lóticos, Índice de Qualidade da Água.

\section{COMPARISON BETWEEN THE WATER QUALITY INDEX (WQI) TO THE PROTOCOL OF RAPID ASSESSMENT OF HABITATS IN THE LISO STREAM IN SÃO SEBASTIÃO DO PARAÍSO, MINAS GERAIS}

\footnotetext{
${ }^{1}$ Engenheiro Ambiental, FESP/UEMG E-mail: alexandre.csouza@hotmail.com

${ }^{2}$ Graduanda em Ciências Biológicas, FESP/UEMG. E-mail: thainadfr@yahoo.com.br

${ }^{3} \mathrm{Dr}^{\mathrm{a}}$ em Ecologia e Recursos Naturais, coordenadora e professora do curso de Ciências Biológicas, FESP/UEMG. E-mail: odilarigolin@yahoo.com.br
} 


\begin{abstract}
In the last decades, studies on conservation and monitoring of habitats is shown in constant and significant growth. New monitoring techniques have been developed and are considered important in scientific circles, just for the ease, speed and accuracy of the results presented. This work had as objective use the Rapid Assessment Protocols, proposed by Callisto et al. (2002), as a tool for visual, fast and qualitative evaluation, aiming to evaluate the structure and functioning of ecosystems with the water quality index (WQI) in order to contribute for the management and conservation. The data collections were made on March 20 and 23 2014, in six sampling sites in the Liso Stream, located in the São Sebastião do Paraíso town, MG. It is noticed that in the Liso Stream there is a strong anthropic action, and with the protocol application was possible to diagnose it as "impacted" according to the final score of all the points studied ranged 26-31. According to the report published by Instituto Mineiro de Gestão das Águas (IGAM) the said stream presented a WQI of 25,1 in the 4th quarter 2013, and in the 1st quarter 2014 presented a WQI of 34,6, however remaining as "bad", which is confirmed in the Rapid Assessment Protocols methodology, showing its efficiency to diagnose lotic environments. Need is still urgent mitigation measures apply to the stream does not worsen further their limnological quality, increasingly aggravating their classification before the IQA.
\end{abstract}

KEY-WORDS: Habitats, Lotic Environments, Water Quality Index.

\title{
COMPARACIÓN ENTRE EL ÍNDICE DE CALIDAD DEL AGUA (ICA) UTILIZANDO EL PROTOCOLO DE EVALUACIÓN RÁPIDA DE LOS HÁBITATS EN EL ARROYO LISO, MUNICIPIO DE SÃO SEBASTIÃO DO PARAÍSO, MINAS GERAIS
}

\begin{abstract}
RESUMEN
En las últimas décadas, los estúdios sobre la conservación y monitoreo de los hábitats muestranse en crecimiento constante y significativo. Nuevas técnicas de monitoreo se han desarrollado y se consideran importantes en los círculos científicos, justo por la facilidad, rapidez y precisión de los resultados presentados. Este estudio tuvo como objetivo utilizar el Protocolo de Evaluación Rápida de la Diversidad de hábitats, propuesto por Callisto et al. (2002), como una herramienta para la evaluación visual, rápida y cualitativa para evaluar la estructura y el funcionamiento de los ecosistemas y comparar con el Índice de Calidad del Agua (ICA), para contribuir con la gestión y conservación de ellos. Las recolectas de datos se llevaron a cabo el 20 y 23 de marzo de 2014, en seis puntos de muestreo en el Arroyo Liso, ubicado en el municipio de São Sebastião do Paraíso, Minas Gerais. Se dio cuenta de que em el Arroyo Liso hay una fuerte acción humana, y com el uso del Protocolo fue posible diagnosticarlos como "impactado" de acuerdo a la puntuación final de todos los puntos estudiados que fueron del 26 al 31. De acuerdo con el informe publicado por el "Instituto Mineiro de Gestão das Águas (IGAM) dijo Arroyo presentó un ICA de 25.1 en el cuarto trimestre de 2013 y en el primer trimestre de 2014 mostró ICA com 34,6, sin embargo, permaneciendo como "malo", que se confirmó utilizando la metodología del protocolo rápido, que muestra su eficacia para diagnosticar ambientes lóticos. Se necesita aún aplicar medidas de mitigadoras urgentes para que el Arroyo no empeore aún más su calidad limnológica, agravando cada vez más su clasificación del ICA.
\end{abstract}

PALABRAS CLAVE: Ambientes lóticos Hábitat, Índice de Calidad del Agua. 


\section{INTRODUÇÃO}

Com a expansão das cidades há aumento da pressão antrópica sobre os corpos hídricos inseridos nessas, pela ocupação do entorno. Isto ocorre, entre outros fatores, devido aos benefícios que estes corpos oferecem, tais como amenização da temperatura, pesca de subsistência e lazer. Entretanto, o uso desordenado do solo e da água, o despejo de resíduos sólidos e a retirada da mata ciliar são alguns dos fatores que atuam na modificação dos ambientes naturais (GOLDANI \& CASSOL, 2008).

A escassez de reservas de água de boa qualidade é decorrente do aumento progressivo da agricultura, mineração e industrialização que causam a poluição de corpos aquáticos e afetam a saúde humana e das comunidades aquáticas (KLEINE \& TRIVINHO-STRIXINO, 2005).

Nas últimas décadas, os ecossistemas aquáticos têm sido alterados em diferentes escalas como consequência negativa de atividades antrópicas. Os rios integram tudo o que acontece nas áreas de entorno, considerando-se o uso e ocupação do solo. Assim, suas características ambientais, especialmente as comunidades biológicas, fornecem informações sobre as consequências das ações do homem. Como consequências observam-se modificações nos cursos e na composição física e química natural dos rios, córregos e lagos, na cobertura vegetal, nas margens, na cor da água, e nos organismos que ali vivem (CALLISTO et al., 2001).

Os autores Silva e Araújo (2003) alertam para o fato de que o consumo de água contaminada por agentes biológicos ou físico-químicos tem sido associado a diversos problemas de saúde, e a garantia do consumo de água potável livre de micro-organismos patogênicos e de substancias e elementos químicos prejudiciais à saúde constituem ação eficaz de prevenção das doenças causadas pela água.

Devido à atual situação em que se encontra a qualidade ambiental das bacias hidrográficas, diversas pesquisas vêm sendo realizadas no Brasil a fim de demonstrar 
a crescente necessidade de conservação da qualidade da água. A Resolução $n^{\circ}$. 357, de 17 de março de 2005, do Conselho Nacional de Meio Ambiente (CONAMA) apresenta os padrões de diversos parâmetros para o enquadramento das águas brasileiras, tornando-se uma referência importante para o monitoramento da qualidade hídrica (BRASIL, 2005).

Com base em Esteves (2011), é importante destacar que o estado de degradação dos recursos hídricos tem revelado a necessidade de se estabelecer métodos de avaliação rápida de sua qualidade ambiental. Nesse contexto, uma ferramenta possível de ser utilizada como ferramenta complementar no monitoramento dos recursos hídricos, são os Protocolos de Avaliação Rápida (PARs), os quais têm a proposta de avaliar, de forma integrada, parâmetros que determinam a qualidade dos condicionantes físicos dos riachos e/ou rios.

Com isso, este trabalho teve como objetivo avaliar a eficiência e praticidade da utilização do Protocolo de Avaliação Rápida de Diversidade de Habitats, no Córrego Liso do Município de São Sebastião do Paraíso - MG, e comparar com o Índice de Qualidade da água estimado pelo Instituto Mineiro de Gestão das Águas - IGAM.

\section{MATERIAIS E MÉTODOS}

\section{1 Área de Estudo}

O estudo procurou explicitar a base metodológica que deu suporte à pesquisa. Trata-se da aplicação do Protocolo de Avaliação Rápida de Diversidade de Habitats, proposto por Callisto et al. (2002), em seis trechos escolhidos aleatoriamente no Córrego Liso do Município de São Sebastião do Paraíso (MG), pertencente à Bacia do Rio Grande e à Sub-bacia do Rio São João, sendo afluente do Rio Santana, principal fonte de captação de água para abastecimento do município.

A escolha dos pontos para a aplicação dos PAR's foi realizada através de visitas ao campo no mês de Março de 2014, a partir de uma análise prévia utilizando 
- Google Earth. A seleção dos trechos analisados levou em consideração a distribuição espacial da rede hidrográfica e a facilidade de acesso, sendo este, um critério determinante para a escolha dos locais no qual foi aplicado o protocolo (RODRIGUES, 2007).

Figura 1 - Imagem de satélite da localização dos 06 Trechos (pontos) em estudo do Córrego Liso do Município de São Sebastião do Paraíso.

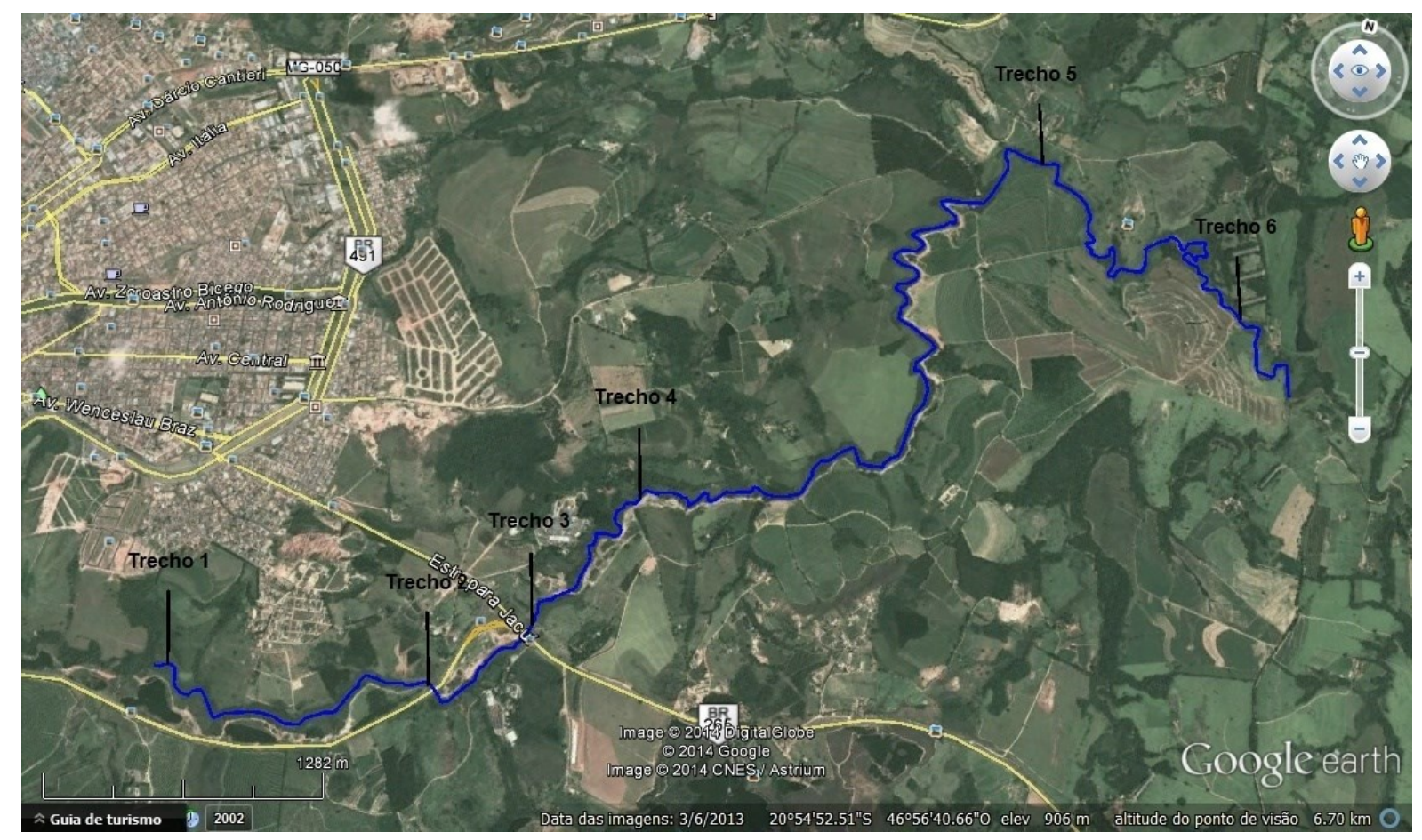

Fonte: Google earth, 2014.

\subsection{Protocolo de Avaliação Rápida (PAR's)}

Os Protocolos de Avaliação Rápida (PAR's) são abordagens modernas para o tradicional uso de indicadores biológicos empregados para avaliação da qualidade ambiental e, dentre as medidas de análises usadas para determinar o grau de impacto empregam-se geralmente à combinação de múltiplas medidas, já que nem todos os parâmetros indicam alterações ambientais com a mesma sensibilidade (MOULTON, 1998; MARQUES \& BARBOSA, 1997; NORRIS \& GEORGES, 1984). 
Foi utilizado o protocolo proposto por Callisto et al. (2002) que é composto por duas partes que avaliam o sistema através de um conjunto de 22 parâmetros distribuídos em categorias descritivas e pontuadas de 0 a 4 na primeira parte (Parâmetros de 1 a 10), e de 0 a 5 na segunda parte (Parâmetros de 11 a 22). A primeira parte do protocolo corresponde a $40 \%$ da pontuação e a segunda parte a $60 \%$.

Os primeiros 10 parâmetros procuram avaliar as características dos trechos e os impactos ambientais decorrentes de atividades antrópicas. A segunda parte do protocolo de Callisto et al. (2002) foi adaptada dos estudos de Hannaford et al. (1997) e buscam avaliar as condições de hábitat e níveis de conservação das condições naturais.

O valor final do protocolo de avaliação será obtido a partir do somatório dos valores atribuídos a cada um dos parâmetros. As pontuações refletem o nível de preservação dos trechos de bacias estudados (Tabela 1) e quando reunidos esses trechos indicam a situação ambiental do trecho.

Tabela 1- Intervalos de pontuação para cada situação ambiental. Protocolo de Avaliação Rápida proposto por Callisto et al. (2002).

\begin{tabular}{c|c}
\hline Pontuação & Situação ambiental \\
\hline 61 a 100 pontos & Natural \\
\hline 41 a 61 pontos & Alterado \\
\hline 0 a 40 pontos & Impactado \\
\hline \multicolumn{2}{r}{ Fonte: SOUZA, 2014 (acadêmico). }
\end{tabular}

As visitas para aplicação dos protocolos e coletas de aspectos físicos como: largura, profundidade e temperatura da água, foram feitas no mês de Março onde tínhamos situação dos dias nublados e parcialmente nublados; ensolarado.

Inicialmente foram sistematizadas as atividades de levantamentos bibliográficos, dados e informações, aquisição de mapas, imagens de satélite da área de estudo e de suporte instrumental composto por um Sistema de Posicionamento 
Global (Modelo - GPSMAP60c5x e Marca Garmin), o qual possibilitou estabelecer a localização geográfica exata de cada local/ponto (trecho) visitado.

Foi usada para a medição da largura do trecho em avaliação uma trena de fibra de vidro de $50 \mathrm{~m}$, assim como, o termômetro para avaliar a temperatura da água; e disco de Secchi para medir a transparência da água.

\section{3 Índice de Qualidade da Água (IQA)}

De acordo com o Instituto Mineiro de Gestão das Águas (IGAM, 2013), a qualidade das águas para abastecimento público é um dos tópicos mais cobrados pela sociedade desde a década de 70, fazendo com que especialistas da área criassem um índice que representasse um padrão de qualidade para determinar o nível de tratamento o qual a água bruta deverá ser submetida. Para determinação desse índice, os cientistas da National Sanitation Foundation (NSF) escolheram nove parâmetros que mais representavam a qualidade da água, atribuíram-lhes pesos (Tabela 3) e instituíram curvas que indicavam a qualidade de cada um e a partir deles determinaram o Índice de Qualidade da Água (IQA) que classifica a qualidade em cinco faixas (excelente, boa, média, ruim e muito ruim) (Tabela 2).

$$
\mathrm{IQA}=\prod_{\mathrm{i}=1}^{\mathrm{n}} \mathrm{q}_{\mathrm{i}}^{\mathrm{w}_{\mathrm{i}}}
$$

IQA: Índice de Qualidade das Águas um número entre 0 e 100;

qi : qualidade do i-ésimo parâmetro, um número entre 0 e 100, obtido da respectiva "curva média de variação de qualidade", em função de sua concentração ou medida e

wi : peso correspondente ao i-ésimo parâmetro, um número entre 0 e 1 , atribuído em função da sua importância para a conformação global de qualidade, sendo: 


$$
\sum_{i=1}^{n} w_{i}=1
$$

n: número de variáveis que entram no cálculo de IQA.

Tabela 2 - Faixas de Classificação conforme a variação do IQA Classificação Faixa de IQA.

\begin{tabular}{l|c}
\hline \multicolumn{1}{c|}{ Classificação Faixa de IQA } & Classificação Faixa de IQA \\
\hline Excelente & $90<$ IQA $\leq 100$ \\
\hline Bom & $70<$ IQA $\leq 90$ \\
\hline Médio & $50<$ IQA $\leq 70$ \\
\hline Ruim & $25<$ IQA $\leq 50$ \\
\hline Muito Ruim & IQA $\leq 25$ \\
\hline
\end{tabular}

Tabela 3 - Parâmetros químicos, físicos e microbiológicos analisados para o cálculo de IQA com seus respectivos valores de pesos e unidades para o calculo.

\begin{tabular}{l|c|c}
\hline \multicolumn{1}{c|}{ Parâmetros } & Unidade & Peso (wi) \\
\hline Temperatura & ${ }^{\circ} \mathrm{C}$ & 0,10 \\
\hline $\mathrm{pH}$ & $\mathrm{mg} / \mathrm{L}$ & 0,12 \\
\hline Oxigênio Dissolvido & $\mathrm{mg} / \mathrm{L}$ & 0,17 \\
\hline DBO & $\mathrm{NMP} / 100 \mathrm{ml}$ & 0,10 \\
\hline Coliformes Termotolerantes & $\mathrm{mg} / \mathrm{L}$ & 0,15 \\
\hline Nitrogênio Total & $\mathrm{mg} / \mathrm{L}$ & 0,10 \\
\hline Fósforo Total & $\mathrm{mg} / \mathrm{L}$ & 0,08 \\
\hline Resíduo Total & UNT & 0,08 \\
\hline Turbidez & & \\
\hline
\end{tabular}

Fonte: CETESB, 2013. 
Os parâmetros físicos, químicos e microbiológicos analisados para a determinação do IQA devem seguir os seguintes métodos: pH - determinado pela leitura do potenciômetro, oxigênio dissolvido (Golterman et al.,1978), nitrogênio total (VALDERRAMA, 1981), fósforo total (STRICKLAND \& PARSONS, 1960), coliformes termotolerantes e demanda bioquímica de oxigênio (APHA, 1998), resíduo total e turbidez de acordo com CETESB (2001).

\section{RESULTADOS E DISCUSSÃO}

Ao final da aplicação do "PAR" nos trechos selecionados foi obtida a média dos resultados, classificando o ambiente como impactado, ou seja, o ambiente sofreu ações que prejudicaram o estado de conservação do habitat, prejudicando/modificando todo o meio em relação a sua condição natural (Quadro 3).

Pode-se constatar que, os resultados dos PARs revelaram que as águas do Córrego Liso, passam por fortes impactos antrópicos decorrentes do uso e ocupação do solo e despejos de efluentes domésticos e industriais que prejudicam a qualidade ambiental do referido córrego. Assim, a Resolução, de 19 de dezembro de 1997, considera impacto ambiental como resultante das alterações que se seguem.

Com base no Quadro - 3 observou-se que, os locais que obtiveram o maior número de impactos foi o trecho 3 com 26 pontos; seguido por 28 pontos atribuídos aos trechos 4 e 6 . Estes valores estão relacionados às intensidades das atividades/ações impactantes desenvolvidas em cada setor.

De acordo com o IGAM (2013) o Índice de Qualidade das Águas (IQA) no $4^{\circ}$ trimestre de 2013 verificou-se a predominância da condição de IQA em vinte e cinco por cento $(25,8 \%)$ nas águas do Estado de Minas Gerais foi considerada Boa, mas no Córrego Liso as condições ainda se encontram ruins, com o valor do IQA em 25,1.

As ocorrências de IQA Médio reduziram de 53,5\% no $4^{\circ}$ trimestre de 2012 para $50,5 \%$ no $4^{\circ}$ trimestre de 2013 . Verificou-se, também a redução das ocorrências 
de IQA Ruim, passando de $26,2 \%$ no $4^{\circ}$ trimestre de 2012 para $22,5 \%$ no $4^{\circ}$ trimestre 2013. Já o IQA Muito Ruim também diminuiu de 1,6\% para 1,2\% (IGAM, 2013).

Baseado nos resultados do Protocolo de Avaliação Rápida e do IQA, pode-se dizer que as avaliações realizadas permitiram detectar prejuízos na conservação ambiental em todos os seis (6) trechos do Córrego Liso, localizado no Município de São Sebastião do Paraíso (MG), tendo como resultado "impactado", enquanto que, para o IGAM o resultado detectado no IQA foi ruim, mas houve uma pequena melhora nos resultados indicando que passaram de muito ruim para ruim. Contudo, verificou que as condições de qualidade muito ruim e ruim vêm prevalecendo ao longo dos anos no Córrego Liso. Embora, pode ser observada uma melhora no ano de 2012 em relação aos resultados apresentados em 2011, uma vez que a frequência de ocorrência do Índice de Qualidade da Água em 75\% das águas passou de muito ruim em 2011 para 50\% em 2012 aponta o relatório (NOGUEIRA, 2013).

Já os resultados obtidos no $3^{\circ}$ trimestre de 2013 , as variáveis físico-químicas, bacteriológicas e dos indicadores IQA, coliformes totais (CT), índice de estado trófico (IET) e densidade de cianobactérias do monitoramento realizado no Estado de Minas Gerais, considera que o Córrego Liso, cujo parâmetro de número 10 não atendeu ao limite legal (violação maior ou igual a 100\%) de Alumínio dissolvido, Cianeto Livre, DBO, Escherichia coli, Ferro dissolvido. Estes resultados provavelmente são devido à existência de curtumes e fábrica de fertilizantes presentes na região de São Sebastião do Paraíso (IGAM, 2013, p. 11- 22).

Contudo, o resultado obtido no $4^{\circ}$ trimestre de 2013 nos mostra uma pequena melhora, uma vez que não houve ocorrência de qualidade "muito ruim". Ainda, assim a qualidade da água precisa de uma grande melhora para poder sair da classificação "ruim", como pode ser observado na Figura 2. 
Figura 2 - Qualidade da água do Córrego Liso do Município de São Sebastião do Paraíso (MG).

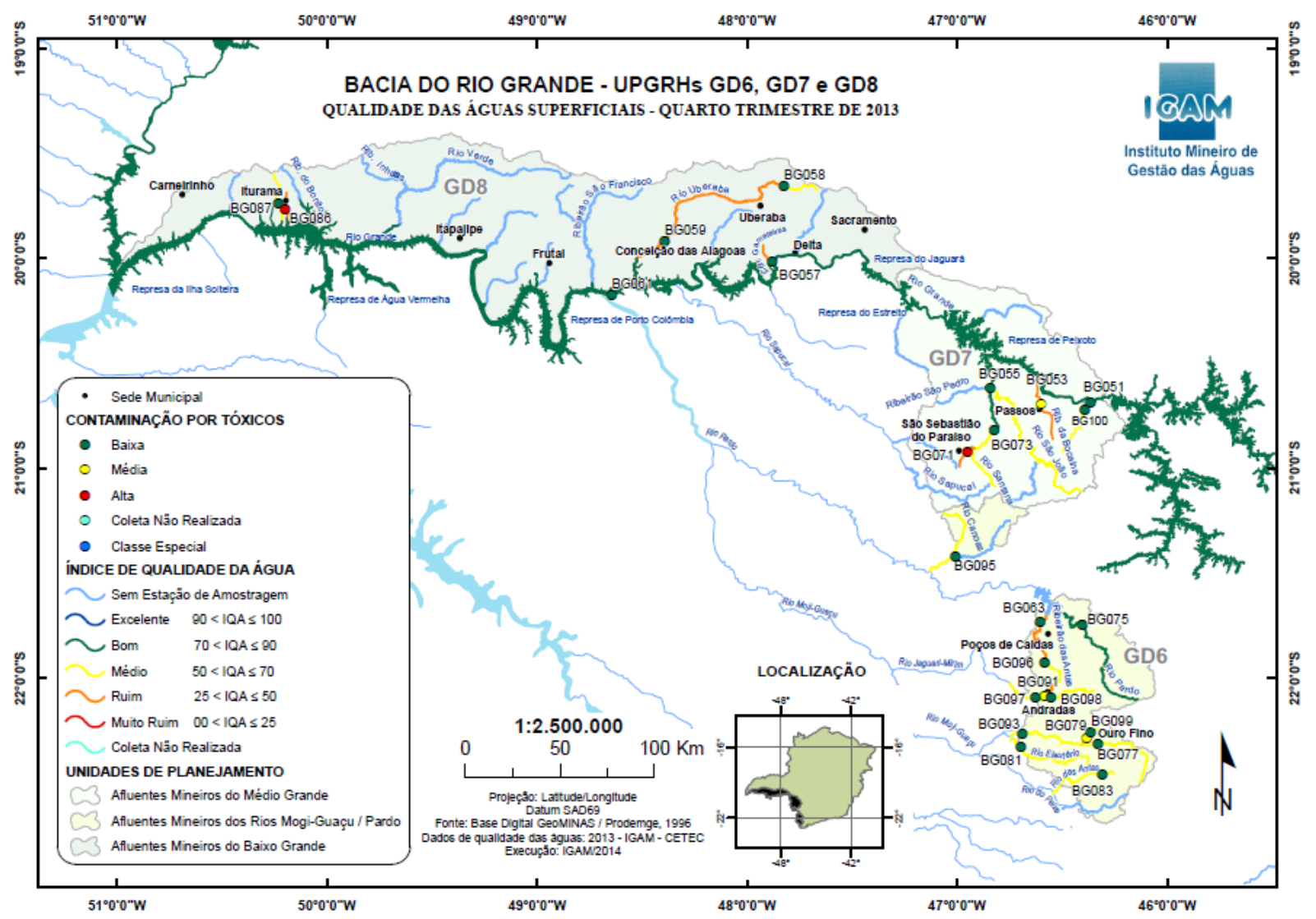

Fonte: Instituto Mineiro de Gestão das Águas (IGAM). Relatório Trimestral. Belo Horizonte, $4^{\circ}$ trimestre de 2013, publicação de março 2014, p. 11.

Segundo Nogueira (2013), também, consta a demanda bioquímica de oxigênio com $100 \%$ das violações, seguido de oxigênio dissolvido com $91 \%$, ferro dissolvido e fósforo total com $87 \%$, cianeto livre com $69 \%$, nitrogênio amoniacal com $61 \%$ e cromo total com $52 \%$ das violações. Estes indicadores refletem os impactos do lançamento dos esgotos domésticos e dos efluentes industriais, mais uma vez comprova que provavelmente do ramo de curtumes de São Sebastião do Paraíso (MG).

Cabe acrescentar que, o Protocolo de Avaliação Rápida de Callisto et al., (2002) propõe analisar o ambiente como um todo, não levando em consideração os parâmetros físico-químicos, mas sim aspectos de observação rápida e análises qualitativa, enquanto que o IQA visa analisar a qualidade da água e não do ambiente 
ao seu redor, estudando somente compostos presentes nela. Pode-se acrescentar o caso do Rio Palmeiras e do Córrego Prata, da cidade de Pratápolis (MG), que através das análises realizadas foi encontrada a presença constante de altas concentrações de nutrientes ( $\mathrm{Cu}, \mathrm{Ni}$ e $\mathrm{Fe}$ ) na água, que é condizente com a alta disponibilidade desses nutrientes junto com o manejo inadequado do solo e o lançamento de efluente industrial e doméstico sem qualquer tipo de tratamento. $O$ uso do solo fora da capacidade de aptidão, o manejo inadequado do solo, o uso indiscriminado de fertilizantes e pesticida, a falta de saneamento e de uma Estação de Tratamento de Efluente (ETE) na região do município, estão comprometendo a qualidade da água do Córrego Prata e do Rio Palmeiras (DUARTE, 2010).

Através dos resultados obtidos, segundo Duarte (2010), pode-se dizer que a qualidade a água no Córrego Prata e no Rio Palmeiras é de boa qualidade, segundo a Resolução n ${ }^{\circ}$ 357, de 17 de março de 2005 do Conselho Nacional de Meio Ambiente (CONAMA), mas apresentou algumas alterações elevadas nos pontos 2 e 3 estudados como por exemplo: $\mathrm{Cu}, \mathrm{Ni}$ e Fe.

Nesse sentido, constatou-se na avaliação da qualidade da água do Córrego Lava Pés no Município de São João Batista do Glória (MG), conforme Rodrigues (2010) que os esgotos domésticos lançados indiscriminadamente, com ou sem tratamento prévio, em ambientes aquáticos afetam profundamente a qualidade da água, provocando drásticas alterações nesses ambientes, como diminuição da quantidade de oxigênio dissolvido, aumento da turbidez, mudanças no $\mathrm{pH}$ e aumento da concentração de nitrogênio e fósforo. Essas alterações na qualidade da água refletem negativamente nas condições ideais para a sobrevivência dos organismos aquáticos. Foi constatado, também, que a urbanização é responsável por profundas modificações na paisagem em que a mata ciliar é retirada, o que causa à exposição das encostas e consequentemente a aceleração dos processos erosivos, com a presença de lixo e resíduos nos corpos d'água, além de alterações no curso normal do rio, assim como a diminuição de diversidade de habitats. 
Com base nos estudos realizados, pode-se dizer que, tanto o Córrego Liso quanto ao Córrego Lava Pés, se encontram eutrofizados pela ação do lançamento de esgoto in natura, além de impactos ambientais causados pela ação antrópica.

De acordo com estudos realizados por Preto (2010) onde o autor realizou análises físicas, químicas e microbiológicas e constatou a presença de coliformes totais e termotolerantes em concentrações tão elevada que não puderam ser contadas. Estes parâmetros podem ser atribuídos ao lançamento de esgoto doméstico sem tratamento prévio; que segundo Hannaford et al. (1997 apud Callisto et al. 2002) contém geralmente mais de 3 milhões de coliformes por $100 \mathrm{ml}$.

\section{CONCLUSÃO}

Coube salientar a importância do monitoramento feito no Córrego Liso pelo IGAM na tentativa de autuar os responsáveis pelo grande e contínuo lançamento de efluentes industriais. Apesar disso, as leis vigentes não conseguem conter boa parte dos praticantes desse crime ambiental, o que condiz do quanto precisamos de leis ainda mais rígidas para não deixarmos nossos ambientes aquáticos a mercê das grandes e pequenas indústrias. Por isso, estudos que contam com a utilização dos Protocolos de Avaliação Rápida (PAR's) reafirmam o mesmo como uma metodologia que se mostra simples e de fácil aplicação, que permite avaliar o impacto não só do ambiente aquático como das áreas de entorno, levando a um diagnóstico mais preciso do ambiente como um todo.

Com isto, este trabalho pode servir como uma base de estudos quanto aos possíveis destinos que podem ser dados a alguns córregos, como o do Córrego Liso e outros mananciais que recebem efluentes de forma inadequada e, levando em conta a importância do uso de metodologias diferentes na discussão dos resultados obtidos na investigação de um ecossistema. 


\section{REFERÊNCIAS}

APHA. American Public Health Association. Standard methods for the examination of water and wastewater. Washignton: APHA, 1998. p.1161.

CALLISTO, M.; MORETTI, M. \& GOULART, M. Macroinvertebrados Bentônicos como Ferramentas para Avaliar a Saúde de Riachos. Revista Brasileira de Recursos Hídricos. Volume 6 n. ${ }^{\circ}$, jan/ mar 2001, 71-82.4.

CALLISTO, M.; FERREIRA, W. R.; MORENO, P.; GOULART, M. \& PETRUCIO, M. Aplicação de um protocolo de avaliação rápida da diversidade de habitats em atividades de ensino e pesquisa (MG-RJ). Acta Limnologica Brasiliensia, 14(1): 91-98. 2002. Disponível em: <http://www.ablimno.org.br/acta/pdf/acta_limnologica_contents1401E_files/Artigo\%2010_14\%281\%29. pdf>. Acessado em: 28 jul. de 2014.

CETESB. Relatório de qualidade das águas interiores do estado de São Paulo 2000. Vol.1. 214p. 2001

DUARTE, D. B. Avaliação da qualidade da água no Córrego Prata e do Rio Palmeiras no Município de Pratápolis (MG). 2010. Artigo de Trabalho de Conclusão de Curso (Bacharel em Engenharia Ambiental) - Fundação de Ensino Superior de Passos. Faculdade de Engenharia de Passos. Passos, MG.

ESTEVES, F. A. Fundamentos da limnologia. 3. ed. Rio de Janeiro: Interciência, 2011.

GOLDANI, J. Z.; CASSOL, R. Ocupação antrópica e socio-ambiental da microbacia do rio Vacacaí- Mirim - RS. Geomática Santa Maria. v. 3, n. 1 e 2, p. 43-55, 2008.

GOLTERMAN, H. L.; CLYMO, RS. \& OHNSTAD, M. A. M. Methods for physical and chemical analysis of freshwaters. Oxford: Blackwell Scientific Publications, 1978. p.213.

HANNAFORD, M.J; BARBOUR, M.T. \& RESH, V.H. Training reduces observer variability in visualbased assessments of stream habitat. Journal North American Benthol. Soc. 16 (4): 853-860. 1997. In: CALLISTO, Marcos; FERREIRA, W. R.; MORENO, P.; GOULART, M. \& PETRUCIO, M. Aplicação de um protocolo de avaliação rápida da diversidade de habitats em atividades de ensino e pesquisa (MGRJ). Acta Limnologica Brasiliensia, 14(1): 91-98. 2002. Disponível em: $<$ http://www.ablimno.org.br/acta/pdf/acta_limnologica_contents1401E_files/Artigo\%2010_14\%281\%29. pdf>. Acessado em: 25 jul. de 2014.

IGAM. Instituto Mineiro de Gestão das Águas. Relatório Trimestral. Belo Horizonte, $3^{\circ}$ trimestre de 2013.

Instituto Mineiro de Gestão das Águas. Monitoramento da qualidade das águas superficiais de Minas Gerais: resumo executivo/Instituto Mineiro de Gestão das Águas. Belo Horizonte: Instituto Mineiro de Gestão das Águas, 2013.

Instituto Mineiro de Gestão das Águas. Monitoramento da qualidade das águas superficiais de Minas Gerais em 2013: resumo executivo / Instituto Mineiro de Gestão das Águas. Belo Horizonte: Instituto Mineiro de Gestão das Águas, 2014.

KLEINE, P.; TRIVINHO-STRIXINO, S. 2005. Chironomidae and other aquatic macroinvertebrates of a first order stream: community response after habitat fragmentation. Acta Limnologica Brasiliensia, 17(1): 91-99. 
MARQUES, M. M. G. S. M. \& BARBOSA, F. A. R. Eficiência de alguns parâmetros da comunidade de macroinvertebrados utilizados na avaliação da qualidade de água. In: VIII SEMINÁRIO REGIONAL DE ECOLOGIA. 1997. São Carlos. Anais...São Carlos: Ed. UFSCAR. 1997. v.7. p.113126.

MOULTON,T.P. Saúde e integridade do ecossistema e o papel dos insetos aquáticos. In: ESTEVES, F. A. \& ESTEVES, A. M. (eds.). Oecologia Brasiliensis - Ecologia de Insetos Aquáticos. Rio de Janeiro. Universidade Federal do Rio de Janeiro. 1998. 5v.p 289-298.

NATIONAL SANITATION FOUNDATION. Water quality index-WQI. Disponível em: <http://www.nsf.org/consumer/earth_day/wqi.asp\#calculating.> Acessado em: 1 ago. de 2014.

NOGUEIRA, R. Qualidade das águas do Córrego Liso ‘evolui para ruim' diz nota do IGAM. Jornal do Sudoeste, São Sebastião do Paraíso (MG). Publicado em 14 dez. 2013. Disponível em: <http://www.jornaldosudoeste.com.br/noticia.php?codigo=4987>. Acesso em: 04 jun. 2014.

NORRIS, R.H. \& GEORGES, A. Analysis and interpretation of benthic macroinvertebrate surveys. In: RESH, V. H. \& ROSENBERG, D. M. (eds.). The ecology of aquatic insects. New York. Praeger Publishers. 1984. p. p235-286.

PRETO, R. J; OLIVEIRA, T. C. T. Previsão da qualidade da água do Córrego Liso, São Sebastião do Paraíso (MG), após instalação da estação de tratamento de efluentes. 2010. Artigo de Trabalho de Conclusão de Curso (Bacharel em Engenharia Ambiental) - Fundação de Ensino Superior de Passos. Faculdade de Engenharia de Passos. Passos, MG.

Resolução $n^{\circ}$. 357, de 17 de março de 2005. Dispõe sobre a classificação dos corpos de água e diretrizes ambientais para o seu enquadramento, bem como estabelece as condições e padrões de lançamento de efluentes, e dá outras providências. Conselho Nacional do Meio Ambiente (CONAMA). Publicada no Diário Oficial da União, nº. 053, de 18/03/2005, p. 58-63.

RODRIGUES, E. Século XXI rumo ao desenvolvimento sustentável e a educação ambiental. Revista Bioética e Educação, Belo Horizonte: Bio Consulte, n. 01, 2007, p. 179-190 (Universidade Federal de Lavras - UFLA).

SILVA, R. C. A.; ARAÚJO, T. M. Qualidade da água do manancial subterrâneo em áreas urbanas de Feira de Santana (BA). Revista Ciência \& Saúde Coletiva, 8(4):1019-1028, 2003. Disponível em: <http://www.usc.br/biblioteca/salusvita/salusvita_v26_n2_2007_art_07.pdf>. Acessado em: 1 ago. de 2014.

STRICKLAND, J. D. H. \& PARSONS, T. R. A manual of seawater analysis. Bulletin of the Fisheries Research Board of Canada, v.125, 1960. p.1-185.

VALDERRAMA, J. C. The simultaneous analysis of total nitrogen and phosphorus in natural waters. Marine Chemistry, v.10, 1981. p.109-122. 


\section{ANEXOS}

Quadro 1 - Protocolo de Avaliação Rápida da Diversidade de Habitats em trechos de bacias hidrográficas, modificado do protocolo proposto pela Agência de Proteção Ambiental de Ohio, EUA (EPA 1987). Obs.: 4 pontos = situação natural; 2 e 0 pontos = situações leve ou severamente alterada Fonte: CALLISTO; FERREIRA; MORENO; GOULART; PETRUCIO. Acta Limnologica Brasiliensia, 14(1): 93, 2002.

\begin{tabular}{|c|c|c|c|}
\hline \multicolumn{2}{|l|}{ Localização: } & \multicolumn{2}{|c|}{ Coordenadas: } \\
\hline \multicolumn{2}{|l|}{ Data Coleta: } & \multicolumn{2}{|c|}{ Hora da Coleta: } \\
\hline \multicolumn{4}{|l|}{ Tempo (Situação do Dia): } \\
\hline \multicolumn{4}{|l|}{ Modo de Coleta: } \\
\hline \multicolumn{4}{|c|}{ Tipo de Ambiente: Córrego ( ) Rio ( ) } \\
\hline \multicolumn{4}{|l|}{ Largura: } \\
\hline \multicolumn{4}{|l|}{ Profundidade: } \\
\hline \multicolumn{4}{|l|}{ Temperatura da Água: } \\
\hline \multirow[t]{2}{*}{ PARÂMETROS } & \multicolumn{3}{|c|}{ PONTUAÇÕES } \\
\hline & 4 Pontos & 2 Pontos & 0 Pontos \\
\hline $\begin{array}{l}1 \text { Tipo de ocupação das } \\
\text { margens do corpo d' água }\end{array}$ & Vegetação natural & $\begin{array}{lcc}\text { Campos } & \text { de } & \text { Pastagem/ } \\
\text { Agricultura/ Reflorestamento }\end{array}$ & Residencial/Comercial//ndustrial \\
\hline $\begin{array}{l}2 \text { Erosão próxima e/ou nas } \\
\text { margens do rio e e } \\
\text { assoreamento em seu leito }\end{array}$ & Ausente & Moderada & Acentuada \\
\hline 3 Alterações Antrópicas & Ausente & $\begin{array}{l}\text { Alterações de origem doméstica } \\
\text { (esgoto, lixo) }\end{array}$ & $\begin{array}{l}\text { Alterações de origem industrial, } \\
\text { urbana (fábricas, canalização, } \\
\text { reutilização do curso do rio) }\end{array}$ \\
\hline 4 Cobertura vegetal no leito & Parcial & Total & Ausente \\
\hline 5 Odor da água & Nenhum & Esgoto (ovo podre) & Óleo/industrial \\
\hline 6 Oleosidade da água & Ausente & Moderada & Abundante \\
\hline 7 Transparência da água & Transparente & Turva, cor de chá forte & Opaca ou colorida \\
\hline $\begin{array}{l}8 \text { Odor do sedimento } \\
\text { (fundo) }\end{array}$ & Nenhum & Esgoto (ovo podre) & Óleo/industrial \\
\hline 9 Oleosidade do fundo & Ausente & Moderado & Abundante \\
\hline 10 Tipo de fundo & Pedras/Cascalho & Lama/ areia & Cimento/canalizado \\
\hline
\end{tabular}


Quadro 2 - Protocolo de Avaliação Rápida de Diversidade de Habitats em trechos de bacias hidrográficas, modificado do protocolo proposto por Hannafordet al. (1997). Obs.: 5 pontos (situação natural); 3, 2 e 0 pontos (situação levemente a severamente alterada). Fonte: CALLISTO; FERREIRA; MORENO; GOULART; PETRUCIO. Acta Limnologica Brasiliensia, 14(1): 94, 2002.

\begin{tabular}{|c|c|c|c|c|}
\hline \multirow[t]{2}{*}{ PARÂMETROS } & \multicolumn{4}{|c|}{ PONTUAÇÕES } \\
\hline & 5 Pontos & 3 Pontos & 2 Pontos & 0 Pontos \\
\hline $\begin{array}{l}11 \text { Tipos de } \\
\text { Fundo }\end{array}$ & $\begin{array}{l}\text { Mais de } 50 \% \text { com habitats } \\
\text { diversificados; troncos } \\
\text { submersos; cascalho ou } \\
\text { outros habitats estáveis. }\end{array}$ & $\begin{array}{l}30 \text { a } 50 \% \text { de habitats } \\
\text { diversificados; } \\
\text { habitats adequados } \\
\text { para a manutenção } \\
\text { das populações de } \\
\text { organismos } \\
\text { aquáticos. }\end{array}$ & $\begin{array}{l}10 \text { a } 30 \% \text { de habitats } \\
\text { diversificados; } \\
\text { disponibilidade de } \\
\text { habitats insuficientes; } \\
\text { substratos } \\
\text { frequentemente } \\
\text { modificados. }\end{array}$ & $\begin{array}{l}\text { Menos que } 10 \% \text { de } \\
\text { habitats diversificados; } \\
\text { ausência de habitats } \\
\text { óbvia; substrato rochoso } \\
\text { instável para a fixação de } \\
\text { organismos. }\end{array}$ \\
\hline $\begin{array}{l}12 \text { Extensão de } \\
\text { Rápidos }\end{array}$ & $\begin{array}{l}\text { Rápidos e corredeiras bem } \\
\text { desenvolvidos; rápidos tão } \\
\text { largos quanto o rio e com o } \\
\text { comprimento igual ao } \\
\text { dobro da largura do rio. }\end{array}$ & $\begin{array}{l}\text { Rápidos com a } \\
\text { largura igual à do rio, } \\
\text { mas com } \\
\text { comprimento menor } \\
\text { que o dobro da } \\
\text { largura do rio. }\end{array}$ & $\begin{array}{l}\text { Trechos rápidos podem } \\
\text { estar ausentes; rápidos } \\
\text { não tão largos quanto o } \\
\text { rio e seu comprimento } \\
\text { menor que o dobro da } \\
\text { largura do rio. }\end{array}$ & $\begin{array}{l}\text { Rápidos ou corredeiras } \\
\text { inexistentes. }\end{array}$ \\
\hline $\begin{array}{l}13 \text { Frequência de } \\
\text { Rápidos }\end{array}$ & $\begin{array}{l}\text { Rápidos relativamente } \\
\text { frequentes; distância entre } \\
\text { rápidos dividida pela } \\
\text { largura do rio entre } 5 \text { e } 7 .\end{array}$ & $\begin{array}{l}\text { Rápidos não } \\
\text { frequentes; distância } \\
\text { entre rápidos dividida } \\
\text { pela largura do rio } \\
\text { entre } 7 \text { e } 15 .\end{array}$ & 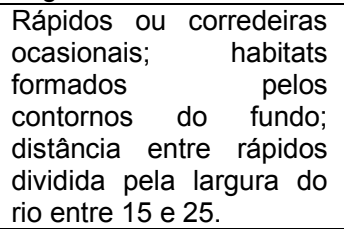 & $\begin{array}{l}\text { Geralmente com lamina } \\
\text { d'água "lisa" ou com } \\
\text { rápidos rasos; pobreza de } \\
\text { habitats; distância entre } \\
\text { rápidos dividida pela } \\
\text { largura do rio maior que } \\
25 \text {. }\end{array}$ \\
\hline $\begin{array}{l}14 \text { Tipos de } \\
\text { Substrato }\end{array}$ & $\begin{array}{lr}\text { Seixos } & \text { abundantes } \\
\text { (prevalecendo } & \text { em } \\
\text { nascentes). } & \end{array}$ & $\begin{array}{l}\text { Seixos abundantes, } \\
\text { cascalho comum. }\end{array}$ & $\begin{array}{l}\text { Fundo formado } \\
\text { predominantemente por } \\
\text { cascalho; alguns seixos } \\
\text { presentes. }\end{array}$ & $\begin{array}{l}\text { Fundo pedregoso ou } \\
\text { lamoso. }\end{array}$ \\
\hline $\begin{array}{l}15 \text { Deposição de } \\
\text { Lama }\end{array}$ & $\begin{array}{l}\text { Entre } 0 \text { e } 25 \% \text { do fundo } \\
\text { coberto por lama. }\end{array}$ & $\begin{array}{l}\text { Entre } 25 \text { e } 50 \% \text { do } \\
\text { fundo coberto por } \\
\text { lama. }\end{array}$ & $\begin{array}{l}\text { Entre } 50 \text { e } 75 \% \text { do fundo } \\
\text { coberto por lama. }\end{array}$ & $\begin{array}{l}\text { Mais de } 75 \% \text { do fundo } \\
\text { coberto por lama. }\end{array}$ \\
\hline $\begin{array}{l}16 \text { Depósitos } \\
\text { Sedimentares }\end{array}$ & $\begin{array}{l}\text { Menos de } 5 \% \text { do fundo } \\
\text { com deposição de lama; } \\
\text { ausência de deposição nos } \\
\text { remansos. }\end{array}$ & $\begin{array}{lr}\text { Alguma evidência } & \text { de } \\
\text { modificação } & \text { no } \\
\text { fundo, } & \\
\text { principalmente } & \text { como } \\
\text { aumento reia } & \text { de } \\
\text { cascalho, areia } & \text { do } \\
\text { lama; 5 a 30\% } & \text { do } \\
\text { fundo afetado; suave } \\
\text { deposição nos } \\
\text { remansos. }\end{array}$ & $\begin{array}{l}\text { Deposição moderada de } \\
\text { cascalho novo, areia ou } \\
\text { lama nas margens; entre } \\
30 \text { e } 50 \% \text { do fundo } \\
\text { afetado; deposição } \\
\text { moderada nos remansos. }\end{array}$ & $\begin{array}{l}\text { Grandes } \\
\text { lama, depósitos }\end{array}$ \\
\hline $\begin{array}{l}17 \text { Alterações no } \\
\text { canal do rio }\end{array}$ & $\begin{array}{l}\text { Canalização (retificação) } \\
\text { ou drenagem ausente ou } \\
\text { mínima; rio com padrão } \\
\text { normal. }\end{array}$ & $\begin{array}{l}\text { Alguma canalização } \\
\text { presente, } \\
\text { normalmente } \\
\text { próxima à construção } \\
\text { de pontes; evidência } \\
\text { de modificações há } \\
\text { mais de } 20 \text { anos. }\end{array}$ & $\begin{array}{l}\text { Algumas modificações } \\
\text { presentes nas duas } \\
\text { margens; } 40 \text { a } 80 \% \text { do } \\
\text { rio modificado. }\end{array}$ & $\begin{array}{l}\text { Margens } \\
\text { acima de } 80 \% \text { do rio } \\
\text { modificado. }\end{array}$ \\
\hline $\begin{array}{l}18 \\
\text { Características } \\
\text { do fluxo da água }\end{array}$ & $\begin{array}{l}\text { Fluxo relativamente igual } \\
\text { em toda a largura do rio; } \\
\text { mínima quantidade de } \\
\text { substrato exposto. }\end{array}$ & $\begin{array}{l}\text { Lamina d'água acima } \\
\text { de } 75 \% \text { do canal do } \\
\text { rio; ou menos de } 25 \% \\
\text { do substrato exposto. }\end{array}$ & $\begin{array}{l}\text { Lâmina d'água entre } 25 \\
\text { e } 75 \% \text { do canal do rio } \\
\text { e/ou maior parte do canal } \\
\text { do substrato nos rápidos } \\
\text { exposto }\end{array}$ & $\begin{array}{l}\text { Lâmina d'água escassa e } \\
\text { presente apenas nos } \\
\text { remansos }\end{array}$ \\
\hline $\begin{array}{l}19 \text { Presença de } \\
\text { mata ciliar }\end{array}$ & $\begin{array}{l}\text { Acima de } 90 \% \text { de } \\
\text { vegetação ripária nativa, } \\
\text { incluindo árvores, arbustos } \\
\text { ou macrófitas; mínima } \\
\text { evidencia } \\
\text { desflorestamento; todas as } \\
\text { plantas atingindo altura } \\
\text { "normal". }\end{array}$ & $\begin{array}{l}\text { Entre } 70 \text { e } 90 \% \text { com } \\
\text { vegetação ripária } \\
\text { nativa;desflorestame } \\
\text { nto evidente mas não } \\
\text { afetando o } \\
\text { desenvolvimento da } \\
\text { vegetação; maioria } \\
\text { das plantas atingindo } \\
\text { altura "normal". }\end{array}$ & $\begin{array}{l}\text { Entre } 50 \text { e } 70 \% \text { com } \\
\text { vegetação ripária nativa; } \\
\text { desflorestamento óbvio; } \\
\text { trechos com solo exposto } \\
\text { ou vegetação eliminada; } \\
\text { menos de metade das } \\
\text { plantas atingindo a altura } \\
\text { "normal". }\end{array}$ & $\begin{array}{l}\text { Menos de } 50 \% \text { da mata } \\
\text { ciliar } \\
\text { desflorestamento nativa; } \\
\begin{array}{l}\text { acentuado. } \\
\text { muito }\end{array}\end{array}$ \\
\hline
\end{tabular}




\begin{tabular}{|c|c|c|c|c|}
\hline $\begin{array}{l}\mathbf{2 0} \text { Estabilidade } \\
\text { das margens }\end{array}$ & 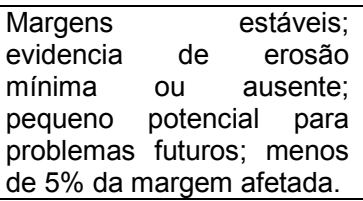 & $\begin{array}{l}\text { Moderadamente } \\
\text { estáveis; pequenas } \\
\text { áreas de erosão } \\
\text { freqüentes; entre } 5 \text { e } \\
30 \% \text { da margem com } \\
\text { erosão }\end{array}$ & $\begin{array}{l}\text { Moderadamente } \\
\text { instável;entre } 30 \text { e } 60 \% \\
\text { da margem com erosão; } \\
\text { risco elevado de erosão } \\
\text { durante enchentes. }\end{array}$ & $\begin{array}{l}\text { Instável; muitas áreas } \\
\text { com erosão; frequentes } \\
\text { áreas descobertas nas } \\
\text { curvas do rio; erosão } \\
\text { óbvia entre } 60 \text { e } 100 \% \text { da } \\
\text { margem. }\end{array}$ \\
\hline $\begin{array}{l}21 \text { Extensão da } \\
\text { mata ciliar }\end{array}$ & $\begin{array}{l}\text { Largura da vegetação } \\
\text { ripária maior que } 18 \text { m.; } \\
\text { sem influência } \\
\text { atividades antrópicas. }\end{array}$ & $\begin{array}{l}\text { Largura da vegetação } \\
\text { ripária entre } 12 \text { e } 18 \\
\text { m; Mínima influência } \\
\text { antrópicas. }\end{array}$ & 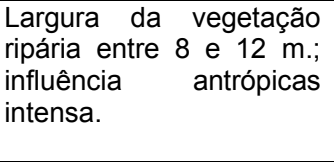 & $\begin{array}{l}\text { Largura da vegetação } \\
\text { ripária menor que } 8 \mathrm{~m} . ; \\
\text { vegetação restrita ou } \\
\text { ausente devido a } \\
\text { atividades antrópicas. }\end{array}$ \\
\hline $\begin{array}{l}22 \text { Presença de } \\
\text { plantas aquáticas }\end{array}$ & $\begin{array}{l}\text { Pequenas macrófitas } \\
\text { aquáticas e/ou musgos } \\
\text { distribuídos pelo leito. }\end{array}$ & $\begin{array}{lr}\text { Macrófitas } & \text { aquáticas } \\
\text { ou algas filamentosas } \\
\text { ou } & \text { musgos } \\
\text { distribuídos no rio; } \\
\text { substrato nom } \\
\text { perifíton. }\end{array}$ & $\begin{array}{l}\text { Algas filamentosas ou } \\
\text { macrófitas em poucas } \\
\text { pedras ou alguns } \\
\text { remansos; } \\
\text { abundante e biofilme. }\end{array}$ & $\begin{array}{l}\text { Ausência de vegetação } \\
\text { aquática no leito do rio ou } \\
\text { grandes bancos de } \\
\text { macrófitas. }\end{array}$ \\
\hline
\end{tabular}

Quadro 3 - Resultados da aplicação do Protocolo de Avaliação Rápida de Diversidade de Habitats (Callisto et al. 2002) no Córrego Liso do Município de São Sebastião do Paraíso, MG. Fonte: SOUZA, 2014 (acadêmico).

\begin{tabular}{|c|c|c|c|c|c|c|}
\hline Parâmetros & Trecho 1 & Trecho 2 & Trecho 3 & Trecho 4 & Trecho 5 & Trecho 6 \\
\hline 1 & 4 & 2 & 2 & 2 & 2 & 2 \\
\hline 2 & 2 & 2 & 0 & 2 & 0 & 2 \\
\hline 3 & 0 & 0 & 0 & 0 & 0 & 0 \\
\hline 4 & 4 & 0 & 0 & 4 & 2 & 4 \\
\hline 5 & 0 & 0 & 0 & 0 & 0 & 0 \\
\hline 6 & 2 & 4 & 4 & 2 & 4 & 4 \\
\hline 7 & 2 & 2 & 2 & 2 & 0 & 0 \\
\hline 8 & 0 & 0 & 0 & 0 & 0 & 0 \\
\hline 9 & 2 & 4 & 4 & 2 & 4 & 4 \\
\hline 10 & 2 & 4 & 2 & 2 & 2 & 2 \\
\hline 11 & 0 & 2 & 2 & 0 & 0 & 0 \\
\hline 12 & 5 & 5 & 5 & 5 & 5 & 2 \\
\hline 13 & 5 & 5 & 5 & 5 & 5 & 2 \\
\hline 14 & 0 & 0 & 0 & 0 & 0 & 0 \\
\hline 15 & 0 & 5 & 0 & 0 & 0 & 0 \\
\hline 16 & 0 & 3 & 0 & 0 & 0 & 2 \\
\hline 17 & 0 & 0 & 0 & 0 & 2 & 2 \\
\hline 18 & 0 & 0 & 0 & 0 & 5 & 0 \\
\hline 19 & 0 & 0 & 0 & 0 & 0 & 0 \\
\hline 20 & 0 & 0 & 0 & 2 & 0 & 2 \\
\hline 21 & 2 & 0 & 0 & 0 & 0 & 0 \\
\hline 22 & 0 & 0 & 0 & 0 & 0 & 0 \\
\hline $\begin{array}{l}\text { Pontuação } \\
\text { Final }\end{array}$ & 30 & 38 & 26 & 28 & 31 & 28 \\
\hline $\begin{array}{c}\text { Situação do } \\
\text { Trecho }\end{array}$ & Impactado & Impactado & Impactado & Impactado & Impactado & Impactado \\
\hline
\end{tabular}

